\title{
Improved techniques for the measurement of consummatory behavior in fishes*
}

\author{
WILLIAM T. WOODARD and M. E. BITTERMAN \\ University of Hawaii, Honolulu, Hawaii 96822
}

\begin{abstract}
Improvements on a technique for the study of consummatory behavior and appetitive conditioning in fishes are described: a target-strike detector module easily incorporated into conventional relay programming equipment, a liquid-food formula highly attractive to goldfish and carp, a reliable Teflon-headed syringe plunger capable of handling gritty foods, and a silent food-pumping system.
\end{abstract}

Holmes and Bitterman (1969) reported a technique for the study of the appetitive behavior of fishes in which a semiliquid food is pumped into a small underwater cup mounted in such a way that the fish's contact with the cup is detected automatically. In the study of consummatory behavior, the cup is baited with food to begin with, and the food is replaced by the pump as it is consumed by the animal. In the study of instrumental conditioning, delivery of food is made contingent on contact with the empty foodcup. We report here certain modifications of the technique which, as long use has shown, make for substantial improvement in reliability and convenience.

The mechanical features of our present response-detection system are much the same as those described by Holmes and Bitterman. A translucent Plexiglas target $(4 \mathrm{~cm}$ diam) with a small cup at its center (see Fig. 1) is illuminated from behind by one of several colored lamps which serve as discriminative stimuli. The target is attached to a stainless steel rod clamped in the needle holder of a crystal phonograph cartridge (Astatic 16), the output of which is connected to a detector circuit which pulses a relay when the target is touched by the animal. An improved detector circuit has been developed which can be mounted and powered like a conventional relay programming module. The circuit (Fig. 2) does not require the bias adjustment of the old circuit (an adjustment which interacted with the sensitivity control and was often subject to drift). Response to low-frequency swaying of the target (a problem with the older circuit) has been minimized by filtering. Potentiometer R 2 controls the duration of a brief period (dead time) which is initiated by a contact and during which the circuit is insensitive to such inputs as target bounce. Monitor Light LED2 provides a visual indication of the dead time. The output is adequate to drive a single $24-\mathrm{V}$ relay of the type used in conventional relay programming systems. Although filters have been incorporated to minimize response to dc powerline transients, inadequately arc-suppressed relays, steppers, or solenoids on the same power buss may occasionally

*This work was supported by Grant MH 23294 from the Public Health Service. produce false responses. This difficulty can be avoided either by reducing the (ample) sensitivity or by checking to see that all components in the system are adequately arc suppressed. A drawing of the printed circuit for Fig. 1 may be obtained from the authors.

A new food formula has been developed which is highly attractive to carp and goldfish, convenient to prepare, and of a stable consistency. Unlike gelatin-based mixtures, it has a consistency which does not change drastically when the food changes from refrigerator to room temperature, and, unlike starch-based mixtures, it requires no cooking which might alter its flavor or nutritional value. The food is prepared according to the following procedure: Mix $30 \mathrm{~g}$ of Longlife Tropical Flake Food, Biorell, Tetramin, or other comparable food with $300 \mathrm{ml}$ of water in a high speed blender for $3 \mathrm{~min}$. Strain the mixture through a No. 40 sieve, return it to the blender set at low speed, and blend for another $3 \mathrm{~min}$ with $3.5 \mathrm{~g}$ of powdered tragacanth slowly added while the blender is operating to avoid clumping. As the tragacanth dissolves, the mixture becomes more viscous and frothy, making it necessary to rid the mixture of air bubbles which would otherwise cause the food quickly to float to the surface when dispensed through the foodcup. Deaerated, the food stays in the cup, and, lacking the compressible bubbles, it does not continue to ooze into the cup as the bubbles expand under the relieved pressure when pumping is terminated. While the mixture can be partially deaerated by allowing it to stand for several hours, the quickest and most satisfactory procedure is vacuum deaeration. Since the mixture will spoil in several days even under refrigeration, our practice is to freeze it in small jars, each holding enough for 2 days, and then to defrost them as needed

Due to the gritty nature of the food, the glass syringe formerly used to pump the food jammed occasionally. The glass plunger has now been replaced with the Teflon-headed plunger shown in Fig. 3. Commercial Teflon plungers tried were not satisfactory due to their compressibility and the resultant oozing of food from the nipple caused by return of the plunger to its normal shape after the syringe pump was turned off. The more 

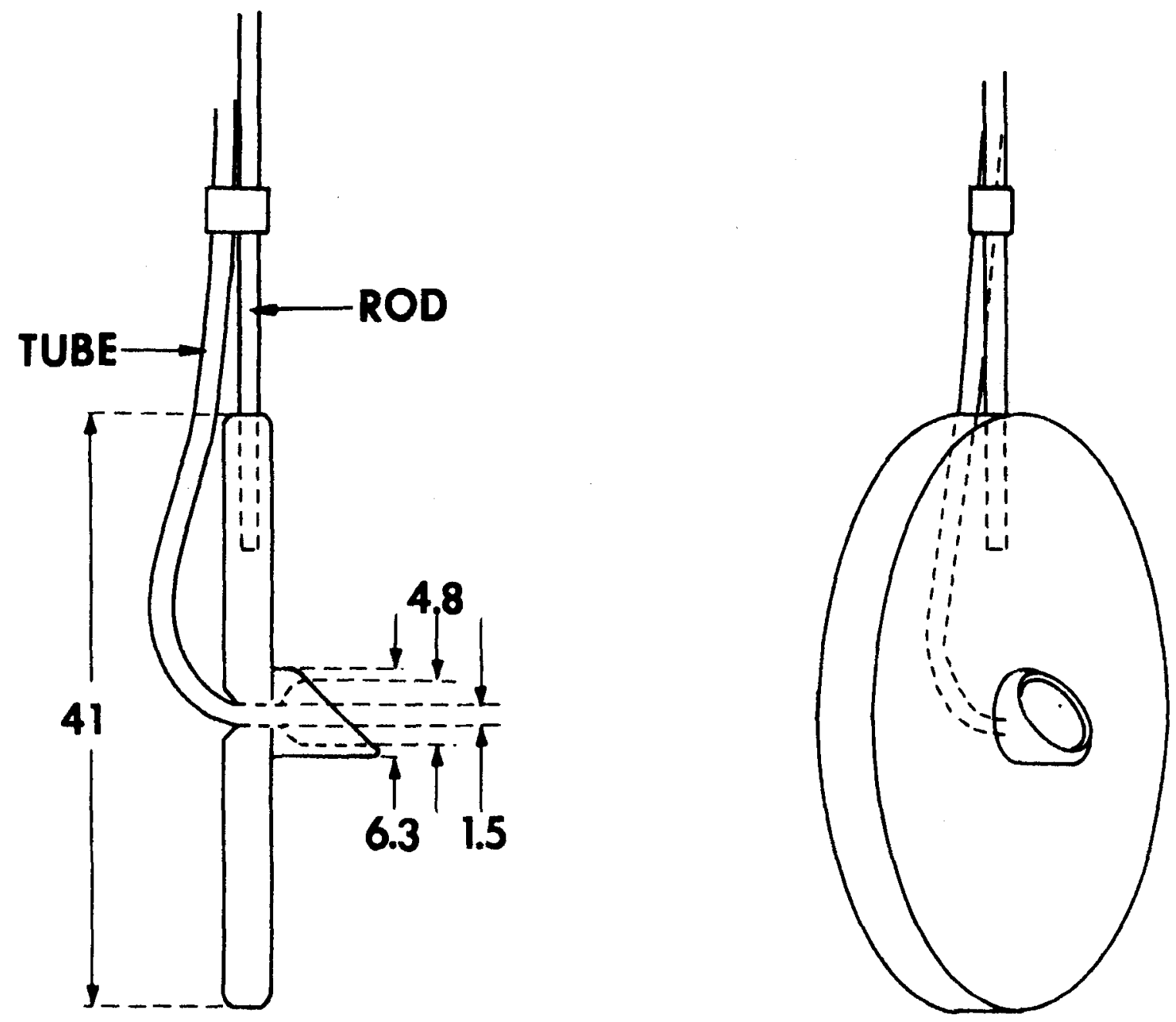

Fig. 1. Target and foodcup. The target is a translucent Plexiglas disk onto which diffuse lights of various colors may be projected from the rear. In the center of the target is the foodcup which is machined from a solid Plexiglas rod and cemented to the target over a hole into which the Teflon food tube is force fitted. The target is suspended from a stainless steel rod clamped in the needle holder of the crystal cartridge, and the food tube is attached to the rod by a short segment of heat-shrinkable tubing as shown. When lines of light are projected on the target as in a line-tilt discrimination, food is introduced into the cup via a channel bored into the target disk along a radius not used in the experiment so that the food channel will not shadow the projected lines. All dimensions are given in millimeters. 

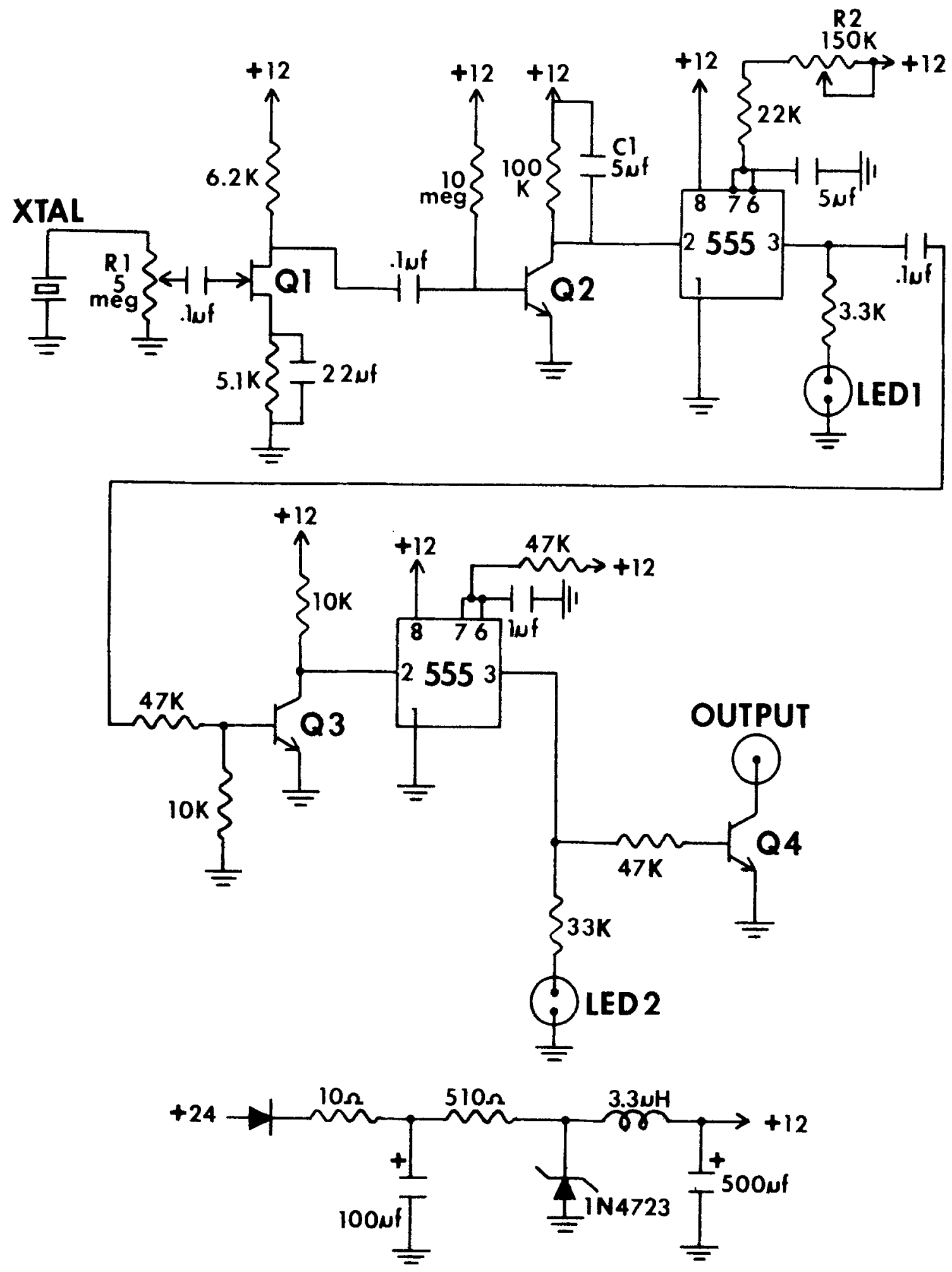

Fig. 2. Response-detec tor circuit. XTAL, Astatic 16 crystal phonograph cartridge; Q1, 2N3819; Q2 and Q3, MPS6566; Q4, $2 \mathrm{~N} 2219$; R1, sensitivity; R2, dead time. The integrated circuits are Signetics 555 timers. If noise problems are not severe, $\mathrm{C} 1$ may be reduced to provide increased sensitivity. 


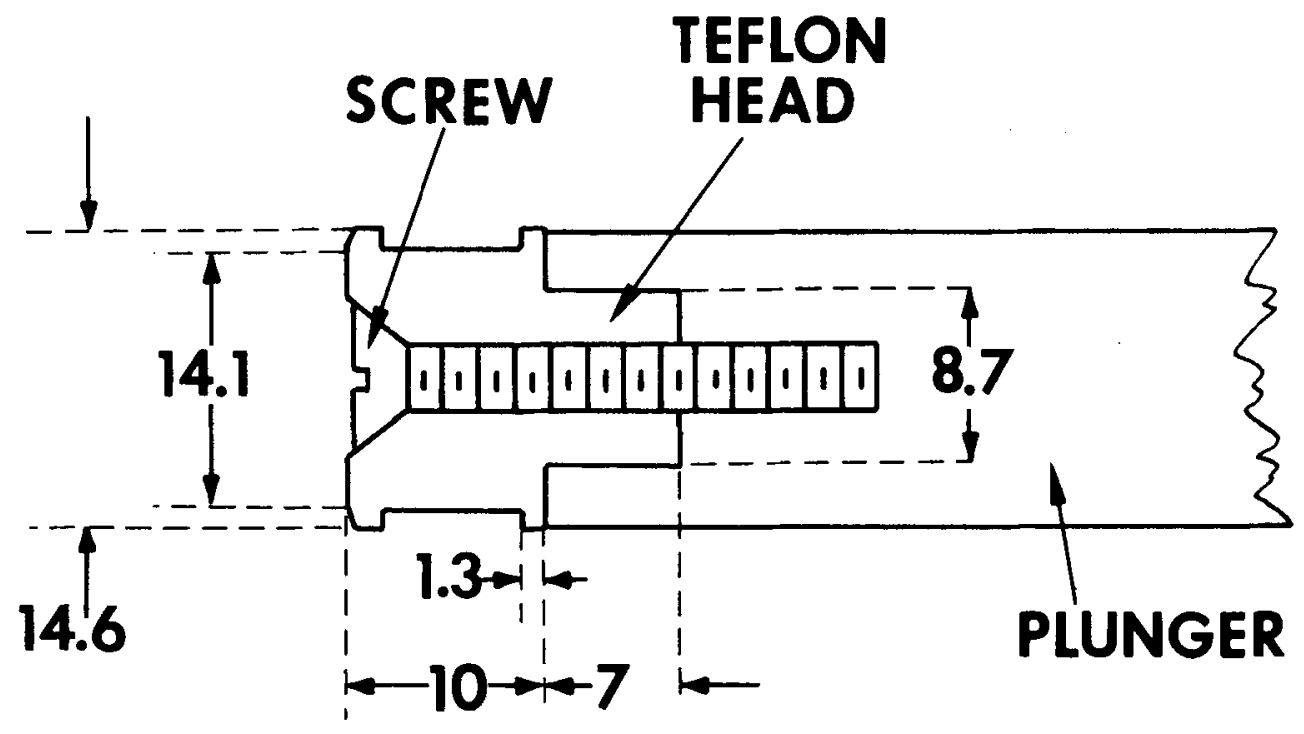

Fig. 3. Plunger. The main body of the plunger is machined from Plexiglas rod to fit loosely in the syringe barrel. The Teflon head (the depth of the ridges are exaggerated for clarity in the drawing) fits just tightly enough to permit slight air leakage under pressure. The final fit is achieved by adjusting the stainless steel screw which expands the head slightly when tightened. The dimensions given (in millimeters) are for a 10-cc (Multifit) syringe.

rigid design shown in Fig. 3 also permits adjustment of the fit of the plunger to the glass barrel-when the screw at the head is tightened, it expands the head slightly and produces a tighter seal.

We now drive the 10-cc food syringe with a Harvard Petipump (Model 1152). Since the pump provides continuous drive as long as its synchronous motor is powered, the amount of food dispensed (approximately $50 \mu \mathrm{l}$ in our experiments with 3.4-in. goldfish) may conveniently be controlled by a pulse former having an adjustable pulse width. Replacement motors of various speeds also may be ordered. Unlike solenoid-driven syringe pumps, the Petipump is silent and the amount of food delivered at each feeding can be programmed readily. For critical applications where oozing of food after switching off the motor must be minimized, the brass sleeve bearings of the Petipump may easily be replaced with bearings machined to closer tolerances, and the spring holding the split nut in contact with the drive screw replaced with a stiffer spring.

\section{REFERENCE}

Holmes, N. K., \& Bitterman, M. E. Measurement of consummatory behavior in the fish. Journal Experimental A naly sis of Behavior, 1969, 12, 39-41.

(Received for publication January 25, 1974; accepted January 30,1974 .) 